\title{
LAGRANGE INTERPOLATION SET ALONG LINEAR PIECEWISE ALGEBRAIC CURVES*
}

\author{
REN-HONG WANG ${ }^{\dagger}$ AND SHAO-FAN WANG W $^{\ddagger}$
}

\begin{abstract}
This paper discusses the Lagrange interpolation problem in continuous bivariate spline spaces over regular triangulations. By using the so-called Lagrange interpolation set along piecewise algebraic curves, we develop a new approach of constructing the interpolation set for continuous spline spaces. We show the property of this set on star region, and construct the interpolation set for continuous bivariate spline spaces over arbitrary triangulations. The construction only depends on the number of points on the piecewise algebraic curve in each cell.
\end{abstract}

Key words. Bivariate spline, Lagrange interpolation set, linear piecewise algebraic curve, interpolation set along piecewise algebraic curves.

AMS subject classifications. 41A05, 41A15, 41A63, 65D05, 65D07.

\section{Introduction}

Let $D$ be a bounded simply connected domain in $\mathbf{R}^{2}$ and let $\Delta$ be a regular triangulation of $D$ with a polygonal boundary, i.e., a finite set of closed triangles such that any pair of triangles intersect only at a common vertex or along a common edge. Let $k, \mu$ be integers with $k>\mu \geq 0$. The space of bivariate splines of degree $k$ and smoothness $\mu$ is defined by

$$
S_{k}^{\mu}(\Delta):=\left\{s \in C^{\mu}(\Delta):\left.s\right|_{\Delta_{i}} \in \mathbf{P}_{k}, \quad i=1, \ldots, T\right\},
$$

where $\Delta_{i}, i=1, \ldots, T$ are all the cells of $\Delta$ and $\mathbf{P}_{k}$ denotes the space of bivariate polynomials of total degree $k$.

We say that a set of points $\mathcal{A}=\left\{Q_{i}, i=1,2, \ldots, d\right\} \subset D$, where $d=\operatorname{dim} S_{k}^{\mu}(\Delta)$, is a Lagrange interpolation set for $S_{k}^{\mu}(\Delta)$ if for any given data $y_{1}, \ldots, y_{d} \in \mathbf{R}$ there exists a unique spline function $s \in S_{k}^{\mu}(\Delta)$, such that

$$
s\left(Q_{i}\right)=y_{i}, \quad i=1, \ldots, d .
$$

We are interested in the Lagrange interpolation problem in the space of bivariate splines, that is, determining the interpolation set for any spline spaces. While in univariate spline spaces, the interpolation set can be characterized by the SchoenbergWhitney condition, it is more difficult to determine the interpolation set in the multivariate case. Davydov et al. showed the characterization of the interpolation set on arbitrary triangulations in [3] by investigating the zero set of bivariate linear spline. Other scholars, e.g., Chui, He, Hecklin, Nürnberger, Schumaker and Wang, did much research on this topic $[1,2,4,7,10]$. Nürnberger and Zeilfelder gave a summary of interpolation methods on bivariate spline spaces in [8].

*Received: April 29, 2008; accepted (in revised version): December 12, 2008. Communicated by Shi Jin.

Project supported by the National Science Foundation of China (Nos. 60533060, 60373093, 10726068, 10801024) and the Innovation Foundation of the Key Laboratory of High Temperature Gas Dynamics, Chinese Academy of Sciences.

${ }^{\dagger}$ Institute of Mathematical Sciences, Dalian University of Technology, Dalian 116085, China (renhong@dlut.edu.cn).

${ }^{\ddagger}$ Corresponding author. Institute of Mathematical Sciences, Dalian University of Technology, Dalian 116085, China (shaofan_dut@163.com). 
We call the zero set

$$
Z(s):=\left\{(x, y): s(x, y)=0, s \in S_{k}^{\mu}(\Delta)\right\}
$$

a piecewise algebraic curve of degree $k$ and smoothness $\mu$ in $\Delta$. By linear algebra, a set of points $\mathcal{A}=\left\{Q_{i}, i=1,2, \ldots, \operatorname{dim} S_{k}^{\mu}(\Delta)\right\}$ is an interpolation set for $S_{k}^{\mu}(\Delta)$ if and only if a non-trivial piecewise algebraic curve $s(x, y)=0$ such that $\mathcal{A} \subset Z(s)$ does not exist. Therefore, the Lagrange interpolation problem is equivalent to discussing the property of piecewise algebraic curves. Our main idea comes from the work proposed by Liang et al. in $[5,6,11]$, who discussed the Lagrange interpolation problem in multivariate polynomial spaces. Wang and Zhu extended this method to consider the interpolation problem in bivariate spline spaces [13]. In this paper, we develop a method to construct the interpolation set for continuous spline spaces, by using the so-called Lagrange interpolation set along piecewise algebraic curves. The paper is organized as follows. In section 2, we introduce the so-called Lagrange interpolation set along piecewise algebraic curves. By using the Bezout theorem for piecewise algebraic curves in section 3, we show an interesting property of the interpolation set along linear piecewise algebraic curves over star regions. A new approach for constructing the interpolation set for bivariate continuous spline spaces over arbitrary triangulations is given in section 4 .

\section{Preliminaries}

Throughout the paper, we use the following important notation. Let $V$ be an interior vertex of a triangulation $\Delta$ and denote $S t(V):=\left\{\Delta_{i} \in \Delta: V \in \Delta_{i}, i=1,2, \ldots, N\right\}$ to be the set of triangles sharing $V$ as a common point, which is called a star region of $V$. Let $p, q$ be two polynomials of positive degrees. If there exists a natural number $k \geq 2$, such that $p^{m} \mid q$ holds for $m=1,2, \ldots, k$, then $p$ is said to be a multiple factor of $q$. We denote $S_{k}^{-1}(\Delta)$ to be the space of piecewise polynomials of degree $k$. We denote $I N(f, g)$ to be the number of intersections between two curves $f=0$ and $g=0$. We denote $\#(A)$ to be the number of elements of a set $A$.

We shall give the definition of the Lagrange interpolation set along piecewise algebraic curves in this section. Before doing this, we need to introduce the so-called WELL piecewise algebraic curve, which plays an important role in our interpolation method.

Definition 2.1. ([13]) Let $k, \mu$ be integers such that $0 \leq \mu \leq k$. Let $q(x, y) \in S_{k}^{\mu}(\Delta)$ be a spline function such that for each cell $\delta \in \Delta$, the polynomial $\left.q\right|_{\delta}$ has no multiple factors, and none of the interior edges lies on the curve $q=0$. Then the curve $q=0$ is called a WELL piecewise algebraic curve (WPAC).

We give the definition and property of the so-called Lagrange interpolation set along a WELL piecewise algebraic curve as follows. In the following Definition 2.2 and Theorem 2.3 we shall let $n, k$ be natural numbers and $\mu$ be an integer such that $0 \leq \mu \leq n-k$. Let $q(x, y) \in S_{k}^{\mu}(\Delta)$ be a spline function such that the curve $q(x, y)=0$ is a WPAC on $\Delta$. Denote $e_{n}^{\mu}(k):=\operatorname{dim} S_{n}^{\mu}(\Delta)-\operatorname{dim} S_{n-k}^{\mu}(\Delta)$ to be the difference between the dimensions of $S_{n}^{\mu}(\Delta)$ and $S_{n-k}^{\mu}(\Delta)$. Let $\mathcal{B}=\left\{Q_{i}, i=1,2, \ldots, e_{n}^{\mu}(k)\right\}$ be a set of distinct points on the WPAC $q=0$.

DEFINITION 2.2. ([13]) If for any spline function $s(x, y) \in S_{n}^{\mu}(\Delta)$ with $s\left(Q_{i}\right)=0$, $i=1, \ldots, e_{n}^{\mu}(k)$, there exists a piecewise polynomial $r(x, y) \in S_{n-k}^{-1}(\Delta)$ such that $s=q r$, then the set $\mathcal{B}$ is called a Lagrange interpolation set along the WPAC $q=0$ with respect to the spline space $S_{n}^{\mu}(\Delta)$. For short, we say $\mathcal{B} \in I_{n, k}^{\mu}(q)$. 
ThEOREM 2.3. ([13]) Let $\mathcal{A}$ be an interpolation set for $S_{n-k}^{\mu}(\Delta)$ such that none of points of $\mathcal{A}$ lies on the WPAC $q=0$. Then $\mathcal{A} \cup \mathcal{B}$ is an interpolation set for $S_{n}^{\mu}(\Delta)$ if and only if $\mathcal{B} \in I_{n, k}^{\mu}(q)$.

REMARK 2.4. If the piecewise algebraic curve $q(x, y)=0$ is not a WPAC, the conclusion is not valid because for any spline function $s(x, y) \in S_{n}^{\mu}(\Delta)$ such that the curve $s=0$ passes through all the points of $\mathcal{A} \cup \mathcal{B}$, there exists a piecewise polynomial $r(x, y) \in S_{n-k}^{-1}(\Delta)$ such that $s=q r$. However $r(x, y)$ is not a spline function of smoothness $\mu$ if one or some interior edges of $\Delta$ lie on the curve $q=0$. In that case we have $r \not \equiv 0$ although it passes through all the points of $\mathcal{A}$, and $\mathcal{A} \cup \mathcal{B}$ is not an interpolation set for $S_{n}^{\mu}(\Delta)$.

Theorem 2.3 tells us that once we have an interpolation set for spline spaces of lower degree, we can construct an interpolation set for spline spaces of higher degree by using the so-called Lagrange interpolation set along a WPAC. At the end of this section, we propose the Bezout theorem and $A F+B G$ theorem for piecewise algebraic curves, which are essential for the proof of our main results (Theorems 3.1 and 3.2).

TheOREM 2.5. ([9]) Let $S t(V)$ be a star region of $V$ and $T$ be the number of cells of $S t(V)$. Denote

$$
B N(m, r ; n, t ; \Delta):=\max _{f, g}\left\{I N(f, g)<+\infty: f \in S_{m}^{r}(\Delta), g \in S_{n}^{t}(\Delta)\right\}
$$

to be the maximum number of intersections between two piecewise algebraic curves in $S_{m}^{r}(\Delta)$ and $S_{n}^{t}(\Delta)$ whose common points are finite. Then $B N(m, 0 ; n, 0 ; S t(V))=m n T$ if $m n T$ is an even number; otherwise, $B N(m, 0 ; n, 0 ; S t(V))=m n T-1$.

THEOREM 2.6. ([12]) Let $S t(V)$ be a star region of $V$ and $T$ be the number of cells of $S t(V)$. Let $m, n, r$ be natural numbers such that $\max \{m, n\} \leq r$ and $m n T$ is an even number. Let $F \in S_{m}^{0}(S t(V)), G \in S_{n}^{0}(S t(V)), H \in S_{r}^{0}(S t(V))$ be spline functions such that two curves $F=0, G=0$ have exactly mnT distinct intersections in $S t(V)$. If the curve $H=0$ passes through all these intersections, then there exist spline functions $A \in S_{r-m}^{0}(S t(V)), B \in S_{r-n}^{0}(S t(V))$, such that $H=A F+B G$.

\section{The Interpolation set along linear piecewise algebraic curve on a star region}

In the following Theorems 3.1, 3.2 and Lemma 3.5 we shall let $S t(V)$ be a star region and $T$ be the number of cells of $S t(V)$ which are denoted by $\Delta_{1}, \Delta_{2}, \ldots, \Delta_{T}$ in a counter-clockwise direction. Denote $l_{i}$ to be the common edge between two adjacent cells $\Delta_{i}$ and $\Delta_{i+1}, i=1,2, \ldots, T$, where $\Delta_{T+1}=\Delta_{1}$. Let $q(x, y) \in S_{1}^{0}(S t(V))$ be a linear spline function such that the curve $q=0$ is a WPAC on $S t(V)$ with $q(V) \neq 0$. Let $k$ be a natural number and $\mathcal{B}$ be a set of $k T$ distinct points on the WPAC $q=0$ such that none of these points lies on any interior edge of $S t(V)$.

We establish the main results of our paper as follows.

TheOREm 3.1. Suppose that $k T$ is an odd integer. Then $\mathcal{B} \in I_{k, 1}^{0}(q)$ if and only if all the sets $\mathcal{B}_{i}=\left.\mathcal{B}\right|_{\Delta_{i}}, i=1,2, \ldots, T$ satisfy one of the following two conditions:

1. $k-1 \leq \#\left(\mathcal{B}_{i}\right) \leq k+1, \quad i=1, \ldots, T$ and the indices $i_{1}<i_{2}<\cdots<i_{\alpha}$ such that $\#\left(\mathcal{B}_{i_{m}}\right)=k-1,1 \leq m \leq \alpha$ and the indices $j_{1}<j_{2}<\cdots<j_{\alpha}$ such that $\#\left(\mathcal{B}_{j_{m}}\right)=k+1$, $1 \leq m \leq \alpha$ satisfy two alternating inequalities: $i_{1}<j_{1}<i_{2}<j_{2}<\cdots<i_{\alpha}<j_{\alpha}$ or $j_{1}<$ $i_{1}<j_{2}<i_{2}<\cdots<j_{\alpha}<i_{\alpha}$, with $1 \leq \alpha \leq[T / 2]$.

2. $\#\left(\mathcal{B}_{i}\right)=k, i=1, \ldots, T$. 
TheOREM 3.2. Let $Q_{i 1}, Q_{i 2}, \ldots, Q_{i t_{i}}$ be all the points of $\mathcal{B}_{i}=\left.\mathcal{B}\right|_{\Delta_{i}}$. Denote $n\left(l_{i}\right), n_{i j}$ to be the slopes of the straight lines $l_{i}, \overline{V Q}_{i j}$ respectively, $j=1,2, \ldots, t_{i}, i=1,2, \ldots, T$. Suppose that $k T$ is an even integer. Then $\mathcal{B} \in I_{k, 1}^{0}(q)$ if and only if one of the following two conditions is satisfied:

1. $k-1 \leq \#\left(\mathcal{B}_{i}\right) \leq k+1, i=1,2, \ldots, T$ and the indices $i_{1}<i_{2}<\cdots<i_{\alpha}$ such that $\#\left(\mathcal{B}_{i_{m}}\right)=k-1,1 \leq m \leq \alpha$ and the indices $j_{1}<j_{2}<\cdots<j_{\alpha}$ such that $\#\left(\mathcal{B}_{j_{m}}\right)=k+1$, $1 \leq m \leq \alpha$ satisfy two alternating inequalities: $i_{1}<j_{1}<i_{2}<j_{2}<\cdots<i_{\alpha}<j_{\alpha}$ or $j_{1}<$ $i_{1}<j_{2}<i_{2}<\cdots<j_{\alpha}<i_{\alpha}$, with $1 \leq \alpha \leq[T / 2]$.

2. $\#\left(\mathcal{B}_{i}\right)=k, i=1,2, \ldots, T$, and the slopes of the straight lines $l_{i}, \overline{V Q}_{i j}, j=$ $1,2, \ldots, k, i=1,2, \ldots, T$ do not satisfy the following equation:

$$
\prod_{i=1}^{T} \prod_{j=1}^{k}\left(n_{i+1, j}-n\left(l_{i}\right)\right)=\prod_{i=1}^{T} \prod_{j=1}^{k}\left(n_{i j}-n\left(l_{i}\right)\right),
$$

where $n_{T+1, j}=n_{1 j}$.

Remark 3.3. When the index $\alpha$ in Condition 1 is equal to 1 , it degenerates into the following condition: there exist two indices $1 \leq i_{1} \leq j_{1} \leq T$ such that $\#\left(\mathcal{B}_{i_{1}}\right)=k-1$, $\#\left(\mathcal{B}_{j_{1}}\right)=k+1$, and $\#\left(\mathcal{B}_{i}\right)=k$ for each $i \neq i_{1}, j_{1}$.

Proof of Theorem 3.1.

Proof. Sufficiency. Suppose that Condition 2 is satisfied. We can check that $\#(\mathcal{B})=k T=\operatorname{dim} S_{k}^{0}(S t(V))-\operatorname{dim} S_{k-1}^{0}(S t(V))$. Let $s(x, y) \in S_{k}^{0}(S t(V))$ be any spline function such that the curve $s=0$ passes through all the points of $\mathcal{B}$. For $i=1,2, \ldots, T$, denote $s_{i}=\left.s(x, y)\right|_{\Delta_{i}}, q_{i}=\left.q(x, y)\right|_{\Delta_{i}}$ to be the polynomials of $s(x, y), q(x, y)$ restricted on the cell $\Delta_{i}$, respectively. Because $k T$ is an odd number and the piecewise algebraic curves $s=0, q=0$ have $k T$ intersections, by Theorem 2.5 they have infinitely many intersections in $\Delta$. Without loss of generality, we assume that $\Delta_{1}$ is one of the cells with infinitely many intersections between them. We consider the following two cases. If the edge $l_{1}$ and the straight line $q_{1}=0$ are not parallel with each other, their intersection is also one of the intersections of two curves $s_{2}=0$ and $l_{2}=0$. Thus the two polynomials $s_{2}$ and $l_{2}$ have $k+1$ common zeros in $\Delta_{2}$, which implies they have infinitely many intersections in $\Delta_{2}$; if $q_{1}=0$ is parallel to the edge $l_{1}$, by the smoothness condition it is also parallel to the straight line $q_{2}=0$. By using a coordinate transformation, we can express these bivariate polynomials $l_{1}, q_{1}, q_{2}, s_{1}, s_{2}$ into the following equations:

$l_{1}=y, \quad q_{1}=c^{\prime} y-c, \quad q_{2}=y-c, \quad s_{1}=\left(c^{\prime} y-c\right) r_{1}(x, y), \quad s_{2}=\left(c^{\prime} y-c\right) r_{1}(x, y)+y r_{2}(x, y)$,

where $c, c^{\prime} \in \mathbf{R}$ with $c \neq 0, c^{\prime}<0, r_{1}(x, y), r_{2}(x, y)$ are two polynomials of degree $k-1$. Condition 2 indicates that the univariate polynomial $s_{2}(x, c)$ has $k$ distinct roots, since the degree of $s_{2}(x, c)$ is $k-1$, we have $s_{2}(x, c) \equiv 0$. Thus we show that in either case, the polynomials $s_{2}$ and $q_{2}$ have infinitely many common zeros on the cell $\Delta_{2}$. Similarly we can prove that two curves $q=0$ and $s=0$ have infinitely many intersections on all the other cells of $S t(V)$. Therefore, there exists a piecewise polynomial $r(x, y) \in$ $S_{k-1}^{-1}(S t(V))$ such that $s(x, y)=q(x, y) r(x, y)$, which proves that $\mathcal{B} \in I_{k, 1}^{0}(q)$.

Suppose that Condition 1 is satisfied, that is, the indices $i_{1}<i_{2}<\cdots<i_{\alpha}$ such that $\#\left(\mathcal{B}_{i_{m}}\right)=k-1$ and the indices $j_{1}<j_{2}<\cdots<j_{\alpha}$ such that $\#\left(\mathcal{B}_{j_{m}}\right)=k+1,1 \leq m \leq \alpha$ satisfy one of the two alternating inequalities. We repeat the former discussion from each pair of two cells $\Delta_{j_{m-1}}, \Delta_{j_{m}}$ to all the others. The approach is the same as before and we omit the details. 
Necessity. Suppose $\mathcal{B} \in I_{k, 1}^{0}(q)$. At first we consider the bound of the number of points of $\mathcal{B}$ on each cell of $S t(V)$. We assume that $\Delta_{1}$ is a cell of $S t(V)$ such that $\#\left(\mathcal{B}_{1}\right) \geq k+2$. Denote $\mathcal{B}=\left\{Q_{1}, Q_{2}, \ldots, Q_{k T}\right\}$ to be all the points of $\mathcal{B}$. Let $s_{1}, s_{2}, \ldots, s_{d}$ be a basis of spline spaces $S_{k}^{0}(S t(V))$ where $d=\operatorname{dim} S_{k}^{0}(S t(V))$. Let $M_{j}=$ $\left(s_{1}\left(Q_{j}\right), s_{2}\left(Q_{j}\right), \ldots, s_{d}\left(Q_{j}\right)\right)$ be the vector whose elements are $s_{i}\left(Q_{j}\right), i=1,2, \ldots, d$, $j=1,2, \ldots, k T$. Then the vectors $M_{1}, M_{2}, \ldots, M_{k T}$ are linearly independent according to the property of the set $\mathcal{B}$. However, for any point $Q_{1} \in \mathcal{B}_{1}$ and any spline function $s(x, y) \in S_{k}^{0}(S t(V))$, if the curve $s=0$ passes through all the points of $\mathcal{B}_{1} \backslash\left\{Q_{1}\right\}$, then $\left.P\right|_{\Delta_{1}}=0$ and $\left.q\right|_{\Delta_{1}}=0$ have infinitely many intersections and the curve $s=0$ also passes the remaining point $Q_{1}$. That means the column vector $M_{1}$ can be generated by $M_{2}, M_{3}, \ldots, M_{k T}$. This is a contradiction, therefore $\#\left(\mathcal{B}_{1}\right) \leq k+1$. Next, we assume that $\Delta_{1}$ is a cell of $S t(V)$ such that $\#\left(\mathcal{B}_{1}\right) \leq k-2$. Because the total number of points of $\mathcal{B}$ is $k T$ and the number of points on each cell is no more than $k+1$, there exist two cells $\Delta_{\beta}, \Delta_{\gamma}$ with $2 \leq \beta<\gamma \leq T$, such that either $\beta=\gamma-1$ or $\#\left(\mathcal{B}_{\beta}\right)=\#\left(\mathcal{B}_{\gamma}\right)=k+1$, $\#\left(\mathcal{B}_{i}\right)=k, \forall \beta<i<\gamma$. For any point $Q_{1} \in \mathcal{B}_{\gamma}$, let $s(x, y) \in S_{k}^{0}(S t(V))$ be a spline function on $S t(V)$ such that $s=0$ passes through all the points of $\mathcal{B} \backslash\left\{Q_{1}\right\}$. By the previous proof, $s=0$ and $q=0$ have infinitely many intersections in each cell $\Delta_{i}, \forall \beta<i<\gamma$. In particular, $s=0$ passes through the point $Q_{1}$. Again, we show that the vector $M_{1}$ can be generated by $M_{2}, M_{3}, \ldots, M_{k T}$, which leads to a contradiction. So we have $k-1 \leq \#\left(\mathcal{B}_{1}\right) \leq k+1$.

Suppose neither of Conditions 1 and 2 is satisfied, i.e., the indices of the cells with $k-1$ points and the indices of the cells with $k+1$ points of $\mathcal{B}$ do not satisfy the alternating inequalities. Then there exist two cells $\Delta_{\beta}, \Delta_{\gamma}$, with $1 \leq \beta<\gamma \leq T$, such that either $\beta=\gamma-1$, or $\#\left(\mathcal{B}_{\beta}\right)=\#\left(\mathcal{B}_{\gamma}\right)=k+1$, \#( $\left.\mathcal{B}_{i}\right)=k, \forall \beta<i<\gamma$. By using a similar approach, we can get a contradiction of linear independence of those vectors $M_{i}, i=1,2, \ldots, k T$. Then we complete the proof.

The following lemma helps to prove Theorem 3.2.

Lemma 3.4. Let $f \in S_{k}^{0}(S t(V)), g \in S_{1}^{0}(S t(V))$ be two spline function such that the curves $f=0, g=0$ are WPACs with exactly $k T$ intersections in $S t(V)$, where $k$ is a natural number, $T$ is the number of cells of $S t(V)$. Suppose $k T$ is an even number. Then there exists a point among these $k T$ intersections such that for any spline function $h \in S_{k}^{0}(S t(V))$, if the curve $h=0$ passes through other $k T-1$ points of these $k T$ intersections, then it also passes through the remaining one.

Proof. Let $f \in S_{k}^{0}(S t(V)), g \in S_{1}^{0}(S t(V))$ be two spline functions such that the curves $f=0, g=0$ have exactly $k T$ intersections in $S t(V)$, and let the set of these $k T$ intersections be denoted by $\mathcal{A}$. Denote $\Delta_{1}, \Delta_{2}, \ldots, \Delta_{T}$ to be all the cells of $\operatorname{St}(V)$ in a counter-clockwise direction, and $l_{i}$ to be the common edge between two cells $\Delta_{i}$ and $\Delta_{i+1}, i=1,2, \ldots, T$. Denote $\Lambda=\left\{h \in S_{k}^{0}(S t(\Delta)): h(Q)=0, \forall Q \in \mathcal{A}\right\}$ to be the collection of spline functions $h$ such that the curve $h=0$ passes through all these $k T$ intersections. By Theorem 2.6, for any spline function $h \in \Lambda$ there exist a constant $A \in$ $\mathbf{P}_{0}$ and a spline function $B \in S_{k-1}^{0}(S t(V))$ such that $h=A f+B g$. Denote $\Lambda_{0}=\{B \in$ $\left.S_{k-1}^{0}(S t(\Delta)): f=B g\right\}$ to be the set of spline functions $B \in S_{k-1}^{0}(S t(\Delta))$ such that $f=$ $B g$. Then $\Lambda$ and $\Lambda_{0}$ are linear spaces and $\operatorname{dim} \Lambda=\operatorname{dim} \mathbf{P}_{0}+\operatorname{dim} S_{k-1}^{0}(S t(V))-\operatorname{dim} \Lambda_{0}$. However, for each $1 \leq i \leq T$ two polynomials $\left.f\right|_{\Delta_{i}},\left.g\right|_{\Delta_{i}}$ have no common factors on each cell $S t(V)$ because they have finite intersections. That means a non-zero spline function in the set $\Lambda_{0}$ does not exist. Therefore we can calculate the dimension of $\Lambda$ as follows: $\operatorname{dim} \Lambda=1+\operatorname{dim} S_{k-1}^{0}(S t(V))=\operatorname{dim} S_{k}^{0}(S t(V))-(k T-1)$. The calculation implies that there exists a point of $\mathcal{A}$ such that for any spline function $h \in S_{k}^{0}(S t(\Delta))$, 
if the curve $h=0$ passes through other $k T-1$ points of $\mathcal{A}$, it also passes through the remaining one. The proof is finished.

To prove Theorem 3.2, we only need to consider the case when the number of the interpolation set along linear WPACs is the same in each cell, which is given in the following lemma.

Lemma 3.5. Suppose $\#\left(\left.\mathcal{B}\right|_{\Delta_{i}}\right)=k, i=1,2, \ldots, T$, and denote $Q_{i 1}, Q_{i 2}, \ldots, Q_{i k}$ to be the points of $\left.\mathcal{B}\right|_{\Delta_{i}}$ in a counter-clockwise direction on the straight line $\left.q\right|_{\Delta_{i}}=0$ with respect to $V$. Suppose that $k T$ is an even integer. Then $\mathcal{B} \in I_{k, 1}^{0}(q)$ if and only if the slopes of these straight lines $l_{i}, \overline{V Q}_{i j}$ do not satisfy equation (3.1).

Proof. Necessity. We assume that the slopes of straight lines $l_{i}, \overline{V Q}_{i j}$ satisfy equation (3.1). Denote $s(x, y) \in S_{k}^{-1}(S t(V))$ to be the piecewise polynomial such that $s(x, y)$ is the product of these straight lines $\overline{V Q}_{i j}, j=1,2, \ldots, k$ restricted on each cell $\Delta_{i}$. That is, $\left.s\right|_{\Delta_{i}}=c_{i} \prod_{j=1}^{k}\left(n_{i j} x-y\right), c_{i} \neq 0, i=1,2, \ldots, T$. We choose $c_{1}=1$,

$$
c_{i+1}=c_{i} \prod_{j=1}^{k} \frac{n_{i j}-n\left(l_{i}\right)}{n_{i+1, j}-n\left(l_{i}\right)}, \quad i=1,2, \ldots, T-1 .
$$

By using the smoothness condition, it follows from equation (3.1) that $s(x, y)$ is a continuous spline function. That means we find a spline function $s(x, y) \in S_{k}^{0}(\Delta)$, such that the curves $s=0$ and $q=0$ have exactly $k T$ intersections on $S t(V)$, which implies $\mathcal{B} \notin I_{k, 1}^{0}(q)$. This proves the necessity.

Sufficiency. Suppose the slopes of these straight lines $l_{i}, \overline{V Q}_{i j}$ do not satisfy equation (3.1). For any $1 \leq \alpha \leq T, 1 \leq \beta \leq k$, we consider equation (3.1) with the slope of $\overline{V Q}_{\alpha \beta}$ replaced by the variable $x$, that is,

$$
C_{1}\left(x-n\left(l_{\alpha-1}\right)\right)=C_{2}\left(x-n\left(l_{\alpha}\right)\right),
$$

where

$$
C_{1}=\frac{1}{n_{\alpha \beta}-n\left(l_{\alpha-1}\right)} \prod_{i=1}^{T} \prod_{j=1}^{k}\left(n_{i+1, j}-n\left(l_{i}\right)\right), \quad C_{2}=\frac{1}{n_{\alpha \beta}-n\left(l_{\alpha}\right)} \prod_{i=1}^{T} \prod_{j=1}^{k}\left(n_{i j}-n\left(l_{i}\right)\right) .
$$

We choose the coordinate system such that the $y$ axis does not pass through the interior of the cell $\Delta_{\alpha}$, and none of points of $\mathcal{B}$ lies on the $y$ axis. Suppose the $y$ axis passes through the interior of $\Delta_{m_{1}}, \Delta_{m_{2}}$, the number of points of $\mathcal{B}_{m_{1}}$ with positive $x$-coordinates and the number of points of $\mathcal{B}_{m_{2}}$ with negative $x$-axis coordinates are $k_{1}, k_{2}$, respectively (see figure 3.1), where $1 \leq m_{1}, m_{2} \leq T, m_{1}, m_{2} \neq \alpha$. By comparing the values of $n\left(l_{i-1}\right), n\left(l_{i}\right)$ and $n_{i j}$, we have $\operatorname{sgn}\left(C_{1}\right)=(-1)^{k-k_{1}+k-k_{2}}, \operatorname{sgn}\left(C_{2}\right)=$ $(-1)^{k T-1-k_{1}-k_{2}}$. As $k T$ is even, $\operatorname{sgn}\left(C_{1}\right)=-\operatorname{sgn}\left(C_{2}\right)$, and we see that equation (3.2) has a unique solution, which is denoted by $n_{\alpha \beta}^{\prime}$. Let $s\left(c_{1}, c_{2}, \ldots, c_{T} ; x, y\right) \in S_{k}^{-1}(S t(V))$ be a piecewise polynomial such that $\left.s\right|_{\Delta_{i}}=c_{i} \prod_{j=1}^{k}\left(n_{i j} x-y\right), \quad i=1,2, \ldots, T, i \neq \alpha$, and $\left.s\right|_{\Delta_{\alpha}}=c_{\alpha}\left(n_{\alpha \beta}^{\prime} x-y\right) /\left(n_{\alpha \beta} x-y\right) \cdot \prod_{j=1}^{k}\left(n_{\alpha j} x-y\right)$, with each $c_{i} \neq 0$. Again, it follows from the smoothness condition that there exist $c_{1}, c_{2}, \ldots, c_{T}$, such that $s(x, y)=$ $s\left(c_{1}, c_{2}, \ldots, c_{T} ; x, y\right) \in S_{k}^{0}(\Delta)$. Denote $Q_{\alpha \beta}^{\prime}$ to be the intersection between two straight lines $n_{\alpha \beta}^{\prime} x-y=0$ and $\left.q\right|_{\Delta_{\alpha}}=0$. Then $Q_{\alpha \beta}^{\prime} \neq Q_{\alpha \beta}$, and two curves $s=0, q=0$ intersect at all the points of $\mathcal{B} \cup\left\{Q_{\alpha \beta}\right\} \backslash\left\{Q_{\alpha \beta}^{\prime}\right\}$. Let $P(x, y) \in S_{k}^{0}(S t(V))$ be any spline function such that $P=0$ passes through all the points of $\mathcal{B}$. According to Lemma 3.4, 
two curves $s=0, q=0$ have exactly $k T$ intersections and $P=0$ passes through $k T-1$ points of them, then $P=0$ also passes through the remaining one, which is the point $Q_{\alpha \beta}^{\prime}$. Therefore, two curves $P=0$ and $q=0$ have $k+1$ intersections on $\Delta_{\alpha}$, and $k$ intersections on other cells of $S t(V)$. Again by using the method in the sufficiency proof in Theorem 3.1, we can prove that $P=0, q=0$ have infinitely many intersections on each cell of $S t(V)$, thus there exists a piecewise polynomial $r(x, y) \in S_{k-1}^{-1}(S t(V))$, such that $P=q r$. So we have $\mathcal{B} \in I_{k, 1}^{0}(q)$, and the lemma is proved. We have finished the proof of Theorem 3.2.

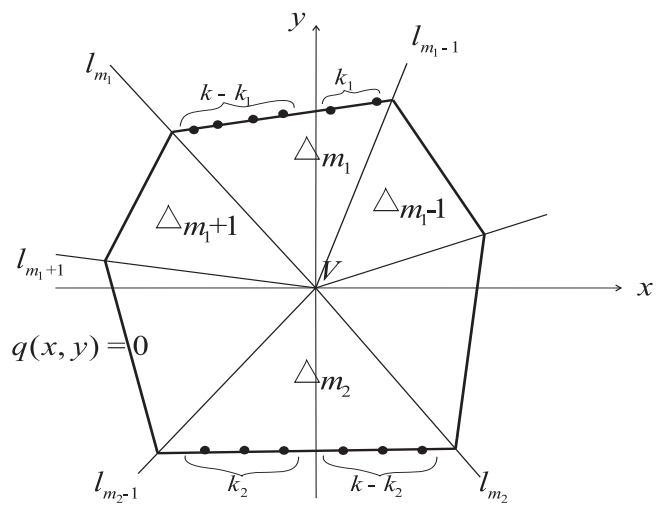

FIG. 3.1. The locations of $\mathcal{B}_{m_{1}}, \mathcal{B}_{m_{2}}$ on the curve $q(x, y)=0$.

Theorems 3.1 and 3.2 tell us whether a set of points is an interpolation set along a linear WPAC on the star region depends on the number of points in cells under most circumstances; when $k T$ is even and the number of points of the set is the same in each cell of the star region, the result also depends on the geometrical property of the set. The reason is that the Bezout theorem for piecewise algebraic curves gives different results under two different circumstances.

\section{Interpolation set for $S_{k}^{0}(\Delta)$ on arbitrary triangulations}

In this section, we propose a method to construct the interpolation set for continuous spline spaces of any degree over arbitrary triangulations. The construction only depends on the number of the points on the linear piecewise algebraic curves within each cell.

In the following theorem, we shall let $\Delta$ be a triangulation and $T$ be the number of cells of $\Delta$. Let $V$ be an interior vertex of $\Delta$ which is adjacent to two boundary vertices of $\Delta$. For $m=1,2, \ldots, k$, let $q_{m}(x, y) \in S_{1}^{0}(\Delta)$ be a spline function such that each curve $q_{m}=0$ is a linear WPAC on $\Delta$ without passing through vertex $V$ and no pair of them has intersections. Let $S t^{1}(V)=S t(V)=\left\{\Delta_{1}, \Delta_{2}, \ldots, \Delta_{n}\right\}$ be the collection of all cells with vertex $V$, and define $S t^{i}(V)$ inductively to be the collection of all cells in $\Delta$ which intersect with $S t^{i-1}(V), i=2,3, \ldots, M:=\max _{v}\{\operatorname{dist}(V, v): v \in V(\Delta) \backslash\{V\}\}$, where $\operatorname{dist}(V, v)$ denotes the smallest number of edges connecting $V$ and $v, V(\Delta)$ denotes the set of all the vertices of $\Delta$ (see figure 4.1). For $2 \leq i \leq M$, denote $\mathcal{C}^{i}$ to be the subset of $S t^{i}(V)$ such that each cell of $\mathcal{C}^{i}$ either intersects at least two interior vertices of $S t^{i-1}(V)$, or intersects with a boundary vertex and an interior vertex of $S t^{i-1}(V)$ and the boundary vertex is adjacent to the interior vertex in the counter-clockwise direction with respect to $V$ (i.e., the counter-clockwise angle from the connecting line 
between $V$ and the interior vertex to the connecting line between $V$ and the boundary vertex, is no greater than $\pi$ ).

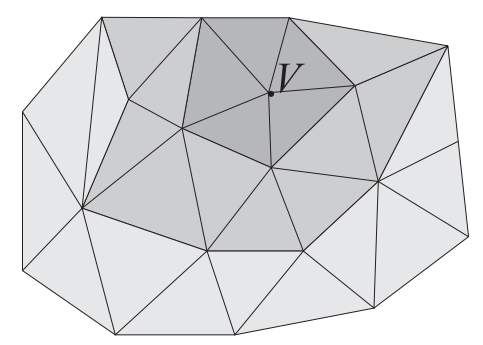

FIG. 4.1. The regions $S t(V), S t^{2}(V)$ and $S t^{3}(V)$ are marked dark gray, bright gray and white.

The construction of interpolation set for $S_{k}^{0}(\Delta)$ is given in the following theorem.

TheOREM 4.1. Suppose $\mathcal{B}$ is a set of points on the curve $\prod_{m=1}^{k} q_{m}(x, y)=0$ without lying on any interior edge of $\Delta$. For each cell $\delta \in \Delta$, denote $b_{\delta}=\left\{\#\left(\left.\mathcal{B} \cap Z\left(q_{m}\right)\right|_{\delta}\right)\right.$ : $m=1,2, \ldots, k\}$ to be the set of numbers of points of $\mathcal{B}$ on curves $q_{m}=0, m=1,2, \ldots, k$ in $\delta$. Suppose that $b_{\delta}$ satisfies the following conditions:

A. $b_{\Delta_{1}}$ is a permutation of $\{2,3, \ldots, k+1\}$;

B. $b_{\delta}$ is a permutation of $\{0,1, \ldots, k-1\}$ if $\delta \in \cup_{i=2}^{M} \mathcal{C}^{i} \bigcup\left\{\Delta_{n}\right\}$;

C. $b_{\delta}$ is a permutation of $\{1,2, \ldots, k\}$ if $\delta \in \Delta \backslash\left(\cup_{i=2}^{M} \mathcal{C}^{i} \cup\left\{\Delta_{1}\right\} \cup\left\{\Delta_{n}\right\}\right)$.

Then $\mathcal{B} \cup\{V\}$ is an interpolation set for spline space $S_{k}^{0}(\Delta)$.

Proof. At first, we check that the number of points of $\mathcal{B} \cup\{V\}$ is equal to the dimension of spline space $S_{k}^{0}(\Delta)$. All the cells of $\Delta$ are divided into $S t^{1}(V), S t^{2}(V), \ldots, S t^{M}(V)$. Denote $V^{I}$ to be the number of interior vertices of $\Delta$, and $I V^{i}$ to be the collection of intersections between $S t^{i-1}(V)$ and $S t^{i}(V)$ which are also the interior vertices of $\Delta, i=2,3, \ldots, M$. It is easy to see that $\#\left(\mathcal{C}^{i}\right)=\#\left(I V^{i}\right)$, which indicates that $\#\left(\sum_{i=2}^{M} \mathcal{C}^{i}\right)=V^{I}-1$. So the number of points of $\mathcal{B}$ is

$$
\begin{aligned}
\#(\mathcal{B}) & =\left(\begin{array}{c}
k+2 \\
2
\end{array}\right)-1+V^{I}\left(\begin{array}{l}
k \\
2
\end{array}\right)+\left(T-V^{I}-1\right)\left(\begin{array}{c}
k+1 \\
2
\end{array}\right)=\left(\begin{array}{c}
k+1 \\
2
\end{array}\right) T-k V^{I}+k \\
& =\operatorname{dim} S_{k}^{0}(\Delta)-1 .
\end{aligned}
$$

Let $s(x, y) \in S_{k}^{0}(\Delta)$ be a spline function such that the curve $s=0$ passes through all the points of $\mathcal{B} \cup\{V\}$. The conclusion is valid if and only if $s$ vanishes on all the cells of $\Delta$. Suppose $\Delta_{1}, \Delta_{2}, \ldots, \Delta_{n}$ are arrayed in counter-clockwise direction with respect to $V$. For $j=1,2, \ldots, n, m=1,2, \ldots, k$, denote $s_{j}=\left.s\right|_{\Delta_{j}}, q_{j m}=\left.q_{m}\right|_{\Delta_{j}}$ to be the polynomials of the spline functions $s, q_{m}$ and $\mathcal{B}_{j}=\left.\mathcal{B}\right|_{\Delta_{j}}, \mathcal{B}_{j m}=\left.\mathcal{B} \cap Z\left(q_{m}\right)\right|_{\Delta_{j}}$ to be the subsets of $\mathcal{B}, \mathcal{B} \cap Z\left(q_{m}\right)$ restricted on cell $\Delta_{j}$, respectively. According to Condition A, there exists a permutation of $\{1,2, \ldots, k\}$, say $\left\{\alpha_{1}, \alpha_{2}, \ldots, \alpha_{k}\right\}$, such that $\#\left(\mathcal{B}_{1 \alpha_{m}}\right)=$ $m+1, m=1,2, \ldots, k$. Then two curves $s_{1}=0, q_{1 \alpha_{k}}=0$ have $k+1$ intersections in $\Delta_{1}$. By using the Bezout theorem, they have infinitely many intersections in $\Delta_{1}$, which implies that there exists a polynomial $r_{1 \alpha_{k}} \in \mathbf{P}_{k-1}$, such that $s_{1}=q_{1 \alpha_{k}} r_{1 \alpha_{k}}$. Because of the property of WPACs, the curve $r_{1 \alpha_{k}}=0$ passes through the vertex $V$ and all the points of $\mathcal{B}_{1} \backslash \mathcal{B}_{1 \alpha_{k}}$. By using the previous discussion repeatedly, we can prove that 
there exists a polynomial $r_{1 \alpha_{1}} \in \mathbf{P}_{0}$, such that $s_{1}=r_{1 \alpha_{1}} \prod_{m=1}^{k} q_{1 \alpha_{m}}$, and the curve $r_{1 \alpha_{1}}=0$ passes through $V$. Because $r_{1 \alpha_{1}}$ is a constant, we obtain that $r_{1 \alpha_{1}} \equiv 0$ and $s_{1} \equiv 0$.

To continue the discussion on $\Delta_{2}$, let $l_{j}$ be the common edge between two cells $\Delta_{j}$ and $\Delta_{j+1}, j=1,2, \ldots, n$, where $\Delta_{n+1}=\Delta_{1}$. It follows from the smoothness condition that there exists a polynomial $p_{2} \in \mathbf{P}_{k-1}$ such that $s_{2}=p_{2} l_{1}$. Repeat the discussion on polynomial $p_{2}$, and it follows from Condition $\mathrm{C}$ that, the curve $p_{2}=0$ has $1,2, \ldots, k$ intersections with these straight lines $q_{21}, q_{22}, \ldots, q_{2 k}$. Thus we obtain that $s_{2} \equiv 0$. The same discussion is applied on $\Delta_{2}, \Delta_{3}, \ldots, \Delta_{n-1}$. For $\Delta_{n}$, the last cell of the star region of $V$, we notice that $s_{n}$ is a polynomial of degree $k$ such that $l_{n-2} l_{n-1} \mid s_{n}$ and the curve $s_{n}=0$ has $0,1, \ldots, k-1$ intersections with those straight lines $q_{n 1}, q_{n 2}, \ldots, q_{n k}=0$ in $\Delta_{n}$; thus $s_{n} \equiv 0$ can be obtained in the same way, and $s$ vanishes on $S t(V)$.
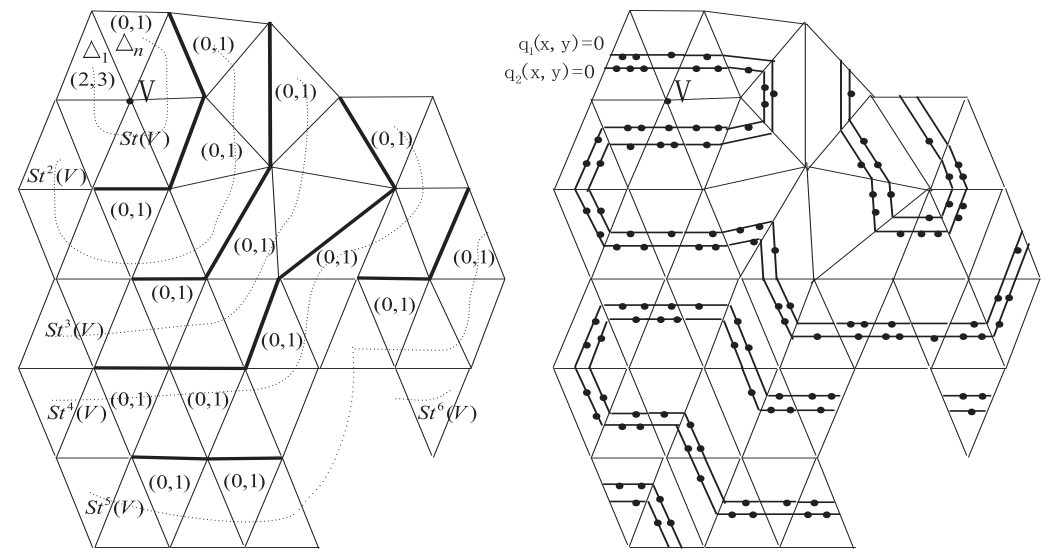

FIG. 4.2. The construction of an interpolation set for $S_{2}^{0}(\Delta)$.

To consider the cells of $S t^{i}(V), i \geq 2$, our main idea is to find an ordering of vertices of $I V^{i}$, the set of intersections between $S t^{i-1}(V)$ and $S t^{i}(V)$ which are also the interior vertices of $\Delta$, such that $s \equiv 0$ can be proved on the star regions of these vertices one by one. We denote $\mathcal{C}_{0}^{i} \subset \mathcal{C}^{i}$ to be the collection of cells which intersect at three interior vertices with $S t^{i-1}(V), 2 \leq i \leq M$. Because each cell of $\mathcal{C}_{0}^{2}$ belongs to the same star region as all the other cells in $S t^{1}(V)$, we can prove that $s$ vanishes on $\mathcal{C}_{0}^{2}$. Moreover, each cell in $\mathcal{C}_{0}^{2}$ intersects with a cell in $S t^{2}(V)$ at two common vertices, and the third vertex is not an intersection between them. We consider the subset of vertices by omitting that vertex from $I V^{2}$. These vertices consist of one or some sequences of adjacent vertices such that the starting and ending points of each sequence are boundary vertices and others are interior vertices. Each edge connecting those interior vertices and the ending point of a sequence is an edge of a cell in $\mathcal{C}^{2}$ (the sub-figure on the left hand side of figure 4.2 shows these edges with boldfaced lines in $\Delta)$. As a result, in the star region of the first interior vertex of each sequence, there is only one cell with $0,1, \ldots, k-1$ numbers of intersections between $s=0$ and $q_{1}, q_{2}, \ldots, q_{k}=0$, which indicates that $s$ vanishes on that star region. Then we repeat the discussion on the star regions of other vertices along the sequence one by one, and prove that $s$ vanishes on $S t^{2}(V)$. Using this approach repeatedly on other star regions of $\Delta$, we can show that $s \equiv 0$ on $\Delta$, which finishes the proof.

An example of constructing the interpolation set for $S_{2}^{0}(\Delta)$ is given in figure 4.2. 
On the left hand side of the figure, we use dashed curves to mark $S t^{i}(V), i=1,2, \ldots, 6$. The cell with $\{2,3\}$ points and the cells with $\{0,1\}$ points are marked, the others contain $\{1,2\}$ points. On the right hand side, $q_{1}(x, y)=0, q_{2}(x, y)=0$ are two linear WPACs with no intersections in $\Delta$. Then the union of the points chosen on two curves $q_{1}=0, q_{2}=0$, and the vertex $V$ is an interpolation set for $S_{2}^{0}(\Delta)$.

Acknowledgement The authors are extremely grateful to the referees for their valuable suggestions and help with the English writing.

\section{REFERENCES}

[1] C.K. Chui, G. Hecklin, G. Nürnberger and F. Zeilfelder, Optimal Lagrange interpolation by quartic $C^{1}$ splines on triangulations, J. Comput. Appl. Math., 216, 344-363, 2008.

[2] C.K. Chui, T.X. He and R.H. Wang, Interpolation by bivariate linear splines, Colloquia. Mathematica and J. Szbadas, (eds.), North Holland, 247-255, 1987.

[3] O. Davydov, M. Sommer and H. Strauss, Interpolation by bivariate linear splines, J. Comput. Appl. Math., 119, 115-131, 2000.

[4] G. Hecklin, G. Nürnberger, L.L. Schumaker and F. Zeilfelder, A local Lagrange interpolation method based on $C^{1}$ cubic splines on freudenthal partitions, Math. Comput., 262, 10171036, 2007.

[5] X.Z. Liang and C.M. Lü, Properly posed set of nodes for bivariate Lagrange interpolation, Approximation Theory IX, C.K. Chui and L.L. Schumaker, (eds.), Vanderbilt University Press, 2, 189-196, 1998.

[6] X.Z. Liang, C.M. Lü and R.Z. Feng, Properly posed set of nodes for multivariate Lagrange interpolation in $C^{s}$, SIAM J. Numer. Anal., 39, 578-595, 2001.

[7] G. Nürnberger, V. Rayevskaya, L.L. Schumaker and F. Zeilfelder, Local Lagrange interpolation with bivariate splines of arbitrary smoothness, Constr. Approx., 23, 33-59, 2006.

[8] G. Nürnberger and F. Zeilfelder, Developments in bivariate spline interpolation, J. Comput. Appl. Math., 121, 125-152, 2000.

[9] X.Q. Shi and R.H. Wang, The Bezout number for piecewise algebraic curves, BIT, 39, 339-349, 1999.

[10] R.H. Wang, Multivariate Spline Functions and their Applications, Science Press/Kluwer Acad. Pub., Beijing/New York, 2001.

[11] R.H. Wang and X.Z. Liang, Approximation of Multivariate Functions, Science Press, Beijing, 1988.

[12] R.H. Wang and C.G. Zhu, Nöther-type theorem of piecewise algebraic curves, Prog. Nat. Sci., 4, 309-313, 2004.

[13] C.G. Zhu and R.H. Wang, Lagrange interpolation by bivariate splines on cross-cut partitions, J. Comput. Appl. Math., 195, 326-340, 2006. 